\title{
Efficient maintenance strategy through System Dynamics
}

\author{
T. Böhm, K. Beck, A. Knaak \& B. Jäger \\ Institute of Transportation Systems, German Aerospace Center, \\ Brunswick, Germany
}

\begin{abstract}
In times when economic efficiency of traffic systems is getting more and more important, it is necessary to have an efficient maintenance strategy, because maintenance costs have been identified as significant cost drivers. This holds true especially for railway signalling in which the long life cycle of elements has a high impact on the costs. In addition, a maintenance strategy is required to fulfil the high standards for availability and safety in the railway sector. While different strategies are available (e.g. corrective or preventive, predetermined or condition based, etc.), each strategy has various options of processed period, duration, costs, impact on the maintained item, etc. This makes it a difficult task to find an efficient maintenance strategy for a system or even a network. The Institute of Transportation Systems has therefore analysed cause-and-effect chains between system condition, availability, maintenance activities, and costs. The identified cause-and-effect chains are modelled with the use of System Dynamics. System Dynamics is a method which has established to analyse and model complex dynamic systems. The developed models do not only enable the user to compare the efficiency of different maintenance activities with each other, but also provide the possibility to determine a combination of these activities leading to an overall system maintenance strategy at a cost minimum through optimisation. This allows infrastructure managers to evaluate their current maintenance program and to determine an economically improved strategy for the future.
\end{abstract}

Keywords: LCC, railway signalling, condition based maintenance, System Dynamics, decision support system. 


\section{Introduction}

Maintenance of railway infrastructure has been identified as a cost driver by infrastructure managers. Cost optimised maintenance strategies have therefore a high impact on the overall economical results of the operation of the railway network [1]. When defining alternative maintenance strategies it is essential to know the cause-and-effect chains between system condition, availability and maintenance measurements. The Institute of Transportation Systems has analysed different elements of railway signalling as part of the railway infrastructure and their maintenance costs throughout their life cycle depending on different maintenance strategies. The identified cause-and-effect chains are implemented in a simulation environment with the use of System Dynamics [2]. The developed model allows optimisation of maintenance strategies for single components of railway signalling elements, the elements themselves and a network of elements.

\section{Modelling maintenance strategies and railway signalling elements}

Railway infrastructure has a long lifetime from 25 up to over 100 years depending on the part of infrastructure and the technology. During this time it is highly required that all parts operate correctly with a high availability to assure save and fluent traffic. Therefore it is necessary to maintain.

\subsection{Evaluated maintenance strategies}

The DIN EN 13306 [3] defines two types of maintenance, the preventive and the corrective maintenance. The first one aims to improve the condition of an item prior to its complete wearout while the latter uses an item until it is worn out and has to be replaced. It is inapplicable for the signalling system in railway, because it puts lives at risk. Hence the research focuses on preventive maintenance.

Preventive maintenance can be predetermined or condition based [3]. The first is typically a set of activities triggered by a time schedule, the latter triggers activities by the wearout. The wearout is diagnosed via manual inspection or sensors. It is also possible to calculate the wearout with statistical methods which allow for the load and the uptime, but it implies that the behaviour of the wearout is known. This would allow to solely trigger maintenance activities by the calculated condition. Since currently there are no adequate or economic diagnosis systems or sensors for most of the signalling elements the three following maintenance strategies are object of investigation:

- Preventive maintenance with predetermined periodic time schedule (time strategy)

- Preventive condition-based maintenance with scheduled inspections to determine the condition (inspection strategy)

- Preventive condition-based maintenance through calculation of condition (condition strategy) 
These strategies are applied to the four most frequently used elements of railway signalling. These are axel counter, switch motor, signal and track circuit.

\subsection{Cause-and-effect chain in maintenance}

The condition of an item can be described as its wearout reserve. The wearout reserve has a range from $100 \%$ to $0 \%$, representing a new item on the one hand and a completely worn out item on the other. Ageing and load increase the wearout, which is decreasing the wearout reserve. A sinking wearout reserve requires enhanced maintenance activity which improves the wearout reserve and lessens the failure rate. The increased wearout reserve reduces the probability of failure and therefore the need for a replacement of an item. A replacement resets the wearout to $100 \%$. The failure rate is associated with the failure. The more failures occur the more activities of fault clearance are required. Fault clearance directly improves the condition of an item or indirectly via replacement. Both increase the costs and the required staff. Figure 1 shows the described generic cause-and-effect chain. It illustrates how the increase of one object affects another one by increasing $(+)$ or decreasing $(-)$ it.

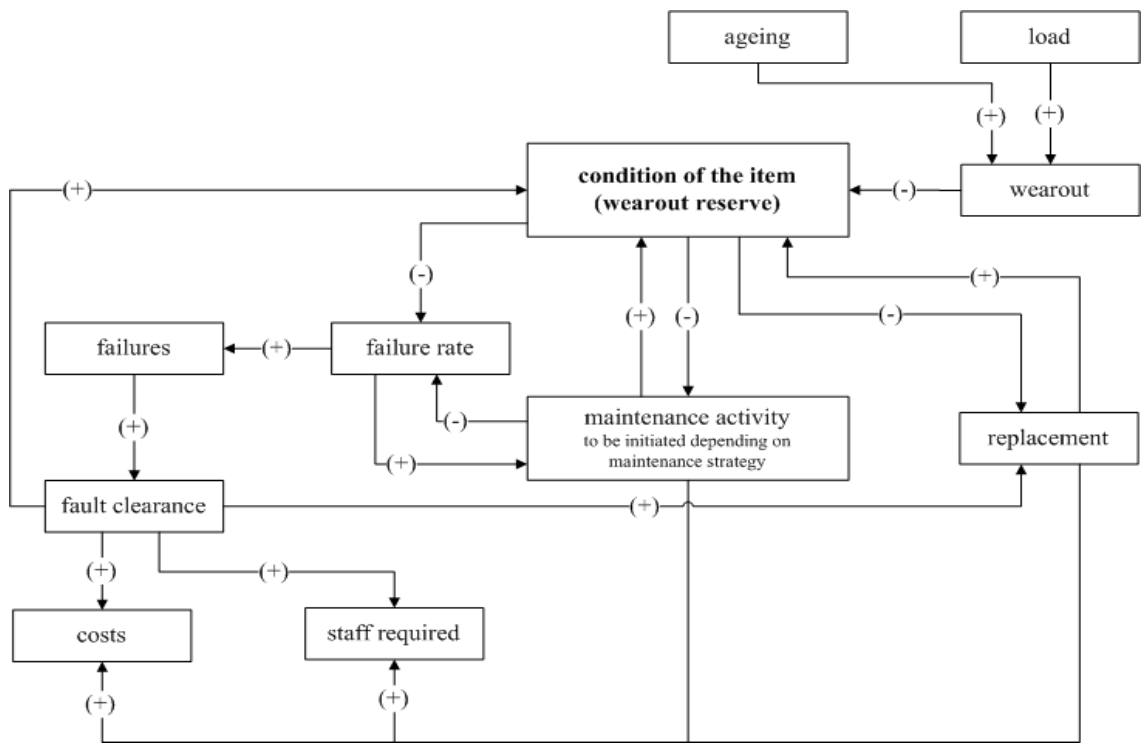

Figure 1: Generic cause-and-effect chain.

\subsection{Basic model in system dynamics}

To find an efficient maintenance strategy it is not enough to qualify the causeand-effect chain, it is also necessary to quantify the association. Once a mathematical equation that describes this association is found, it is possible to simulate the chronological sequence of maintenance costs. 
System Dynamics is a method to qualitatively and quantitatively describe dynamic associations. It was developed by J.W. Forrester of the Massachusetts Institute of Technology at the end of the 1950s [4]. The software AnyLogic ${ }^{\circledR}$ is a tool which enables the building of executable models with System Dynamics as well as simulating these models. Such a simulation requires an adequate mathematical description of the cause-and-effect chain. Therefore it is necessary to make some assumptions.

The first assumption is that the numbers for load and ageing are known. Applied to the signalling items, load means the number of vehicles crossing or the uptime. The number of vehicles crossed is applied in case of a mainly mechanical item. The uptime is used to model a mainly electrical item. Ageing means the duration of time since an item has been installed. To model the wearout reserve the equation of Abeln et al. [5] was adapted as shown in eqn. (1). It computes the wearout reserve (WR) as the summation of load (1), the ageing (a), and the improvement through maintenance (i). The load is one (representing an unused item) minus the wearout coefficient $\left(\mathrm{k}_{1}\right)$ multiplied with the total number of vehicles crossing $\left(\mathrm{cv}_{\text {total }}\right)$ minus the number of vehicles crossing at the last replacement ( $\left.\mathrm{cv}_{\text {lastRep }}\right)$. In case of an electric item the number of crossing vehicle is substituted by the uptime. The ageing is one minus the Euler's number times the ageing coefficient $\left(\mathrm{k}_{\mathrm{a}}\right)$, which is multiplied with the time $(\mathrm{t})$ minus the time at the last replacement ( $\left.\mathrm{t}_{\text {lastRep }}\right)$. The coefficients $\mathrm{k}_{1}$ and $\mathrm{k}_{\mathrm{a}}$ can be understood as the damage done with one crossing vehicle and the reciprocal of the life time of the unused item.

$$
W R=l+a+i=1-k_{l} *\left(c v_{\text {total }}-c v_{\text {last } R \mathrm{e} p}\right)+1-e^{k_{a}^{*\left(t-t_{\text {last Rep }}\right)}}+i
$$

The second assumption is made for the failure rate $(\lambda)$. The failure rate is the stochastic value expressing how often an item fails. Usually manufactures specify the more common MTBF, which is determined through stochastic methods or tests. Per definition, the MTBF is the reciprocal of the failure rate for items with constant failure rate, like electrical items [3]. The weakness of this is that it does not allow for the fact of ageing nor wearout. Both are relevant for mechanical items. The Weibull Distribution is a function often used to represent aging and load of items in their failure rate. The Problem with the Weibull Distribution is the correct adjustment of the parameters $\alpha$ and $\beta$ and its integral. Both parameters are usually unknown and the solution of the integral is an integral, what makes it difficult to automatically solve it in a simulation.

A way to use the MTBF as a given value and allow for ageing and load without the problems of Weibull Distribution is to define the failure rate considering MTBF and wearout reserve as shown in eqn. (2). In this way a new item with a wearout reserve of $1.0(100 \%)$ has the original failure rate, whereas the same older item has a higher chance of a fault. Though it leaves room for improvement, it is a simple and working approach to start with.

$$
\lambda=\frac{1}{M T B F^{*} W R}
$$

Figure 2 shows the key elements of the basic model build in AnyLogic ${ }^{\circledR}$ (the actual model includes more programming elements to store and display data of 


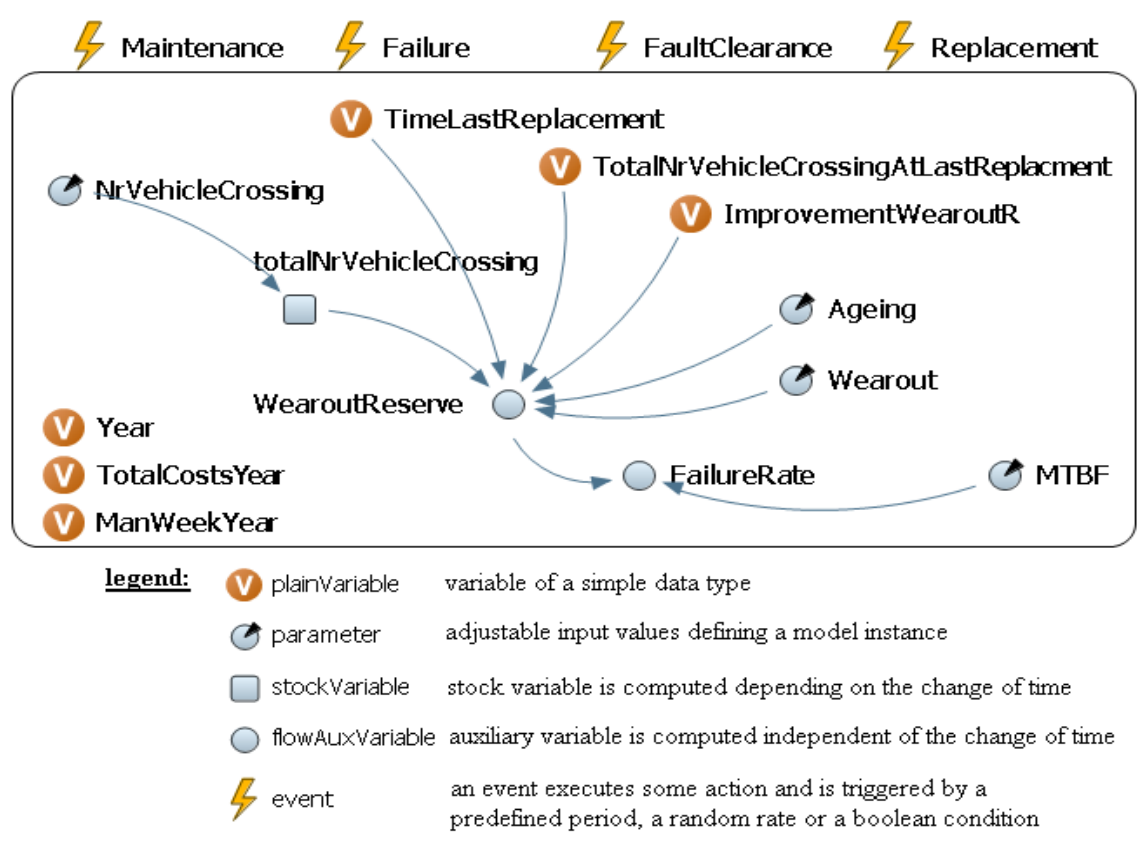

Figure 2: Basic system dynamics model (key elements) of a mechanical item.

the simulation). Associations that are described above can be found in the centre (the functions are included in the source code of the model). All kind of variables (plain, stock, flow auxiliary variable and parameter) are continuously computed during each time step of the simulation. For actions that have no continuous execution an event is used. This is the case for maintenance, failure, fault clearance and replacement. The events have the following triggers:

- Maintenance is either scheduled periodically or dependent on the condition described through the wearout reserve. It depends on the chosen maintenance strategy. The event is improving the wearout reserve of an item with the value of $i$, which is taken from the defined maintenance activity.

- Failure is triggered with a rate which is distributed exponentially. The mean is the failure rate. Thus, failures appear randomly. The lower the wearout reserve is the higher the failure rate and the higher the probability of a failure (see eqn. (2)).

- Fault clearance follows a failure event that sets the condition for the fault clearance event true.

- Replacement is either triggered by a wearout reserve below a predefined level or by the failure clearance event.

Every event is causing costs and man power. This is displayed with the plain variables TotalCostsYear and ManWeekYear. 
The described basic model allows the user to simulate the behaviour (regarding costs, staff required failures, replacements, etc.) of any item over the time.

\subsection{Object oriented methods for modelling a system}

The basic model, as described in the previous section, represents any item. It is the first step to model a system composed of items and even of a network composed of systems. By doing so, it allows to determine an efficient overall strategy or strategy mix. The use of the object oriented development method, as possible with the Java-based AnyLogic ${ }^{\circledR}$, offers a way of how to achieve this goal. The software enables the user to define classes. Each class represents a number of objects holding the same attributes. It is also possible to design classes that are composed of other classes. In addition to the basic model of a component, other classes are needed for the maintenance activity, the system, and the network.

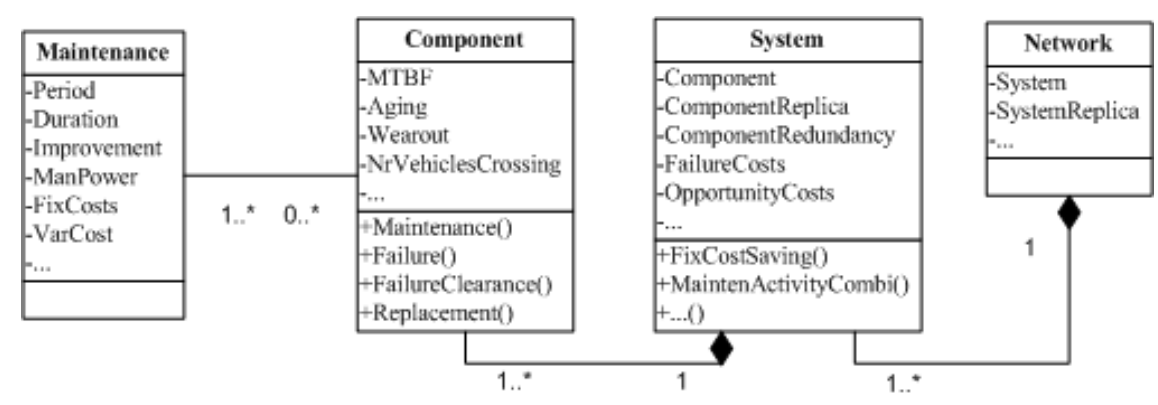

Figure 3: Class diagram of the modeled aspect.

Figure 4 shows the class diagram of the modelled aspects. Therein the component is associated with the maintenance class. The attributes and operations of Component correspond to the parameters and events in Figure 3. The parameters or attributes of Maintenance are the period of time in which the activity is processed (or the level of wearout reserve in case of a condition based strategy), the duration of the activity, the improvement of wearout reserve, different types of cost, etc. This is the place where the difference between the maintenance strategies is set. Every maintenance class attribute is used to define which strategy (see 2.1) is applied and how much it affects the results.

The connection between the classes Component and System means that a component belongs to exactly one system, whereas a system has at least one component. The attribute component defines which component is part of a system. The next two attributes define how often this component is replicated and how many redundancies do exist of it. Due to the possibility of redundant components, failure costs are applied to the system, because a failed component does not necessarily mean a failed system. The same holds true for the opportunity costs, e.g. rail replacement traffic, railway works, rail redirection, 
etc. MaintenActivityCombi is a function that verifies if maintenance activities of some components can be done at the same time to save expenses. Network is a class which is more or less analogous to the class of a system.

\subsection{Model as instrument of strategy evaluation}

After the cause-and-effect chain has been qualified (see 2.2) and quantified, the basic model has been described (see 2.3), and the methods for building the model have been introduced (see 2.4) only one last step is left to an efficient maintenance strategy. This step is the simulation and the optimisation.

Before simulating it is necessary to define components (instances of the class Component), as well as systems and networks by setting the parameters. Also some maintenance activities have to be set. The simulation time can be a number of time steps, a start and stop date using the calendar, or an infinite time. Basically, the simulation is just the running of the model with costs, required staff, number of failures, etc. as a result. It depends on the prior set parameters of the classes. This alone does not give an optimised strategy, especially since it is not meaningful, because some things like failures are random.

At this point the optimisation feature of AnyLogic ${ }^{\circledR}$ is very helpful. It allows an individual number of repeated simulations and an automatic parameter variation. For example, the optimisation is set with one maintenance activity which is periodically done in a range between 10 and 30 weeks and improves the wearout reserve by $20 \%$ at the first time. The parameter variation step for the period could be 0.5 weeks with 200 replications. Then the optimisation would start with a maintenance activity period of 10 weeks, simulate 200 times, and return the average values of costs, failures, etc. Afterwards it simulates 200 times with a 10.5 week period. The average values are compared to the one before and stored if they are better. The 'better' is set prior the optimisation by defining an objective function. In the same way the optimisation continues until the results of a 30 week period are on hand. The output of the completed optimisation is, for instance, the cost optimised period for this component regarding its behaviour. This optimisation applied first to components, then to systems, and at last to a network leads to an efficient maintenance strategy.

\section{Simulation and optimisation of the models}

After the previous section explained how the model is build and used, this section will present the results of an example. It is not intended to determine which strategy is the best to use in general, nor is it the goal to say which wearout reserve level is the best to maintain the gear of a switch motor. This heavily depends on the given parameters. Moreover it is the goal to show that it is possible to find the most cost-efficient strategy for a particular object.

\subsection{Strategy evaluation of a component}

The power supply of a track circuit has been chosen for a comparison between the three strategies for a component. In all of the three cases all parameters of the 
component and the maintenance activities are equal except the ones characterising the maintenance strategy, which are:

- The period of the maintenance activity for the time strategy. It is 12 and 24 months for a small and an extensive activity. Both are independently processed from the actual condition of the component.

- The inspection strategy has an inspection period of 12 months. The small or extensive activity is processed if the inspection reveals a wearout reserve level between $88 \%$ and $76 \%$ or below $76 \%$.

- In case of the condition strategy the small activity is triggered at a wearout reserve level of $88 \%$, the extensive activity at $76 \%$.

The values for period are taken from the corporate policy of the DB AG which specifies maintenance activities for railway signalling [6]. The wearout reserve level is selected reasonably, though it might differ from the ones chosen in real maintenance programs. Due to the fact that failures produce extra high costs, an efficient strategy is mostly going hand in hand with little failures. In this example, with a simulated time of 1200 weeks (about 25 years), the following results are retrieved from simulation. At a similar number of failures the condition strategy has produced $76 \%$ less costs than the time strategy and $67 \%$ less than the inspection strategy. This makes the condition strategy the most efficient strategy in this example.

It may be arguable that there is not always a possibility to choose a strategy, because an inspection does not give the exact condition or the condition behaviour is unknown. This is true, but if all required facts are known it is possible to evaluate how much a different strategy or different activities can contribute to a higher economic efficiency of maintenance processes.

\subsection{Strategy evaluation of systems}

Assuming one of the three strategies is given for a system, a goal could be to reduce costs of maintenance activities. An option is to alter the period or wearout reserve level at which an activity is processed. This could decrease the number of activities during the life cycle of a component and hence its costs. In addition, it might be possible to alter the periods, so that activities of different components of a system can be combined to save fix cost. This could be achieved using the optimisation of the activity's period.

Table 1: $\quad$ Parameter variation for system optimisation.

\begin{tabular}{|l|l|l|l|l|}
\hline Strategy & Altered param. & Lower boundary & Upper & Step \\
\hline time & period of all act. & $\mathrm{Sv}-4[\mathrm{MW}]$ & $\mathrm{Sv}+4[\mathrm{MW}]$ & $1[\mathrm{MW}]$ \\
\hline inspection & inspection period & $\mathrm{Sv}-4[\mathrm{MW}]$ & $\mathrm{Sv}+4[\mathrm{MW}]$ & $1[\mathrm{MW}]$ \\
\hline condition & $\begin{array}{l}\text { wearout reserve } \\
\text { level of all act. }\end{array}$ & $\begin{array}{l}\mathrm{Sv}_{\mathrm{x}}-\left(\mathrm{wl}_{\mathrm{x}+1}-\right. \\
\left.\mathrm{Wl}_{\mathrm{x}+2}\right) * 0.1\end{array}$ & $\begin{array}{l}\mathrm{Sv}_{\mathrm{x}}+\left(\mathrm{wl}_{\mathrm{x}}-\right. \\
\left.\mathrm{Wl}_{\mathrm{x}+1}\right) * 0.1\end{array}$ & 0.01 \\
& \multicolumn{2}{|l}{} \\
\hline
\end{tabular}

As in the strategy comparison, all parameters regarding the behaviour of the model are equal. Only one parameter of each activity is altered in the way described in section 2.5. Table 1 contains the altered parameters for each 
strategy. Therein the lower and upper boundary for the parameter variation is the starting value $(\mathrm{sv}) \pm$ four model weeks $(\mathrm{MW})$, respectively the wearout reserve level (wl). The index $\mathrm{x}(\mathrm{x}=1 . . \mathrm{n})$ stands for a certain wearout reserve level with $\mathrm{wl}_{\mathrm{x}=1}=1.0, \mathrm{wl}_{\mathrm{x}=\mathrm{n}}$ for a security level, and $\mathrm{wl}_{\mathrm{x}=2 \ldots \mathrm{n}-1}$ for the number of actual maintenance activities, each triggered by its wearout reserve level. Every combination of each parameter step is repeated ten times (100 iterations each) to lower the effect of randomly processed failures. As described above, this is done for every component and every system to retrieve average values for costs over the life span of 1200 model weeks. Table 2 shows the result of the parameter optimisation regarding a cost minimum inspection period (inspection strategy). All other strategies have been processed analogously.

Table 2: $\quad$ Period of inspections prior and after the optimisation.

\begin{tabular}{|l|l|r|r|}
\hline Sys tem & Component & \multicolumn{2}{|c|}{ ins pection s trategy (period of ins pection) } \\
& & 24 & {$[\mathrm{MW}]$ before } \\
\hline \multirow{5}{*}{ Signal } & screen & 48 & 27 \\
& LED & 24 & 50 \\
\hline \multirow{5}{*}{ Switch motor } & three-phase motor & 24 & 20 \\
\cline { 2 - 4 } & contacts & 96 & 22 \\
\cline { 2 - 4 } & gear & 24 & 93 \\
\cline { 2 - 4 } & rod & 24 & 20 \\
\hline Track counter & contacts & 24 & 28 \\
\cline { 2 - 4 } & junction box & 24 & 28 \\
\cline { 2 - 4 } & proces sing device & 48 & 28 \\
\cline { 2 - 4 } & power supply & 24 & 50 \\
& insulated rail joint & 24 & 27 \\
\cline { 2 - 4 } & track relay & & 27 \\
\hline
\end{tabular}

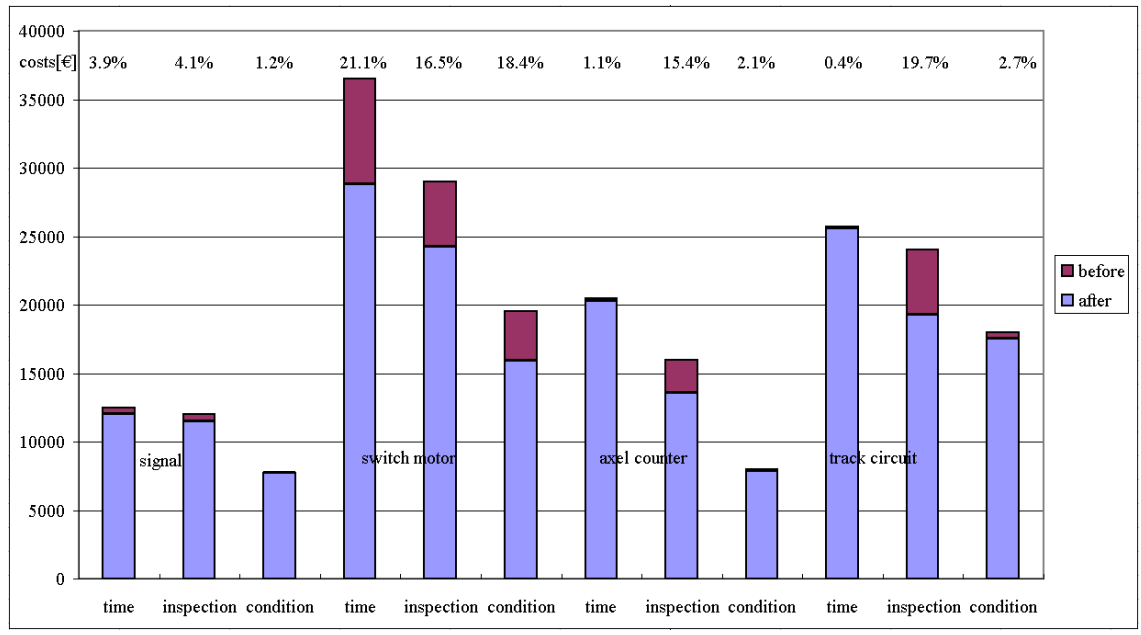

Figure 4: Results of the optimisation. 
An optimisation of the four evaluated elements of railway signalling has shown more or less significant improvements for all strategies in each of the modelled systems. Especially for the switch motor costs have been reduced about $21 \%$ for the time strategy, $16 \%$ for the inspection strategy, and $18 \%$ for the condition strategy. The relatively high reduction can be explained with the fact that a switch motor is a cost intensive system. Therefore the decrease of activities has a higher impact on the cost than in other low cost systems. Figure 4 summarises the costs prior and after the optimisation.

\section{Conclusions and perspectives}

The paper has described the cause-and-effect chains for maintenance strategies and how they are modelled with the use of System Dynamics in AnyLogic ${ }^{\mathbb{R}}$. It has also been shown that with the application of object oriented methods it is possible to build complex models of systems and networks. The models can be simulated and optimised. This enables maintenance managers to determine an efficient maintenance strategy. Though the significance of simulation respectively optimisation result depends on realistic input values, the examples have shown that the chosen approach is capable to improve a maintenance program. The results also indicate the advantages of a condition based strategy, which has shown lower cost in most of the cases. Though it lacks of appropriate techniques to monitor the condition of railway signalling equipment, industry and research have recognised this potential and will therefore search to solve the problem.

The future work of the Institute of Transportation Systems will be to evaluate the approach with real life data in cooperation with an infrastructure operator. Also it is planned to improve mathematical significance of the model, i.e. through the appliance of the Weibull Distribution. But this is more of an enhancement of the working models that have shown success so far.

\section{References}

[1] Kynast, P \& Röpke, B., Diskussion der Instandhaltungswerte in der Leitund Sicherungstechnik, Signal + Draht (93), Hamburg, S. 5-10, 2001.

[2] Sterman, J. D., Business Dynamics - systems thinking and modelling for a complex world, Boston, 2000.

[3] DIN EN 13306:2001-09, Maintenance terminology, Trilingual version, 2001.

[4] Schmidt, D., Strategisches Management komplexer Systeme, Peter Lang: Frankfurt am Main, Bern, New York, Paris, pp. 107-108, 1992.

[5] Abeln, G., Hartmann, T. \& Pitz, V., Zustandsmodelle für ein integriertes Instandhaltungsmanagement von 123-kV-Leistungsschaltern. FGH / ETGFachtagung Diagnostik elektrischer Betriebsmittel, Berlin, pp. 14, 2002.

[6] DB AG, Konzernrichtlinie 892, LST-Anlagen montieren und instand halten, 892.9303-892.9306, 1998-2006. 